\title{
Lost in Translation: Building interpreter confidence and skill in facilitating palliative care conversations
}

\author{
Janet L. Abrahm, MD ${ }^{1}$, Jessica Goldhirsch, LCSW, MSW, MPH ${ }^{2}$
}

${ }^{1}$ Dana-Farber Cancer Institute, Psychosocial Oncology and Palliative Care, Boston, MA, USA

${ }^{2}$ Brigham and Women's Hospital, Social Work, Boston, MA, USA

\section{Introduction}

Diversity of language and culture can create both linguistic and cultural barriers that can result in health care disparities. In the US, for example, while over 300 languages are spoken, the English language and Western health care culture often bump up against the languages and cultures of patients and their families. The need for effective navigation of language and cultural barriers is no more urgent than in the field of palliative care, health care that emphasizes effective communication and attention to the medical, psychosocial and spiritual needs of patients and families coping with serious illness and even end-of-life. Palliative care clinicians can overcome linguistic and cultural barriers by integrating professional interpreters into their interdisciplinary teams. For successful integration and teamwork, however, both parties must be trained.

\section{Objectives}

Improve interpreter confidence in and attitudes towards participating in palliative care family meetings, including both the "pre-meeting" before the encounter, and meetings that include difficult issues facing patients and families, such as breaking bad news and end-of-life discussions.

\section{Methods}

We held six monthly sessions, each with defined curricula and readings, each facilitated by an experienced palliative care social worker and physician. Topics included: Negotiating a preencounter meeting; family meetings in palliative care; accuracy and palliative care terminology; family meetings with goals of care and code status discussions; cultural mediation role plays. Preand post-course evaluations of all participants in attitudes and confidence were done.

\section{Results}

Figure I shows the significant changes in interpreter attitudes in the eleven domains assessed. Figure II shows the highly significant improvement in confidence in palliative care conversations in the thirty interpreter participants.

\section{Conclusions}

Facilitated and well-structured dialogues, including didactics, discussions and roleplaying caused highly significant improvements in interpreter attitudes and confidence in facilitating palliative care conversations.

Figure 1: Attitudes

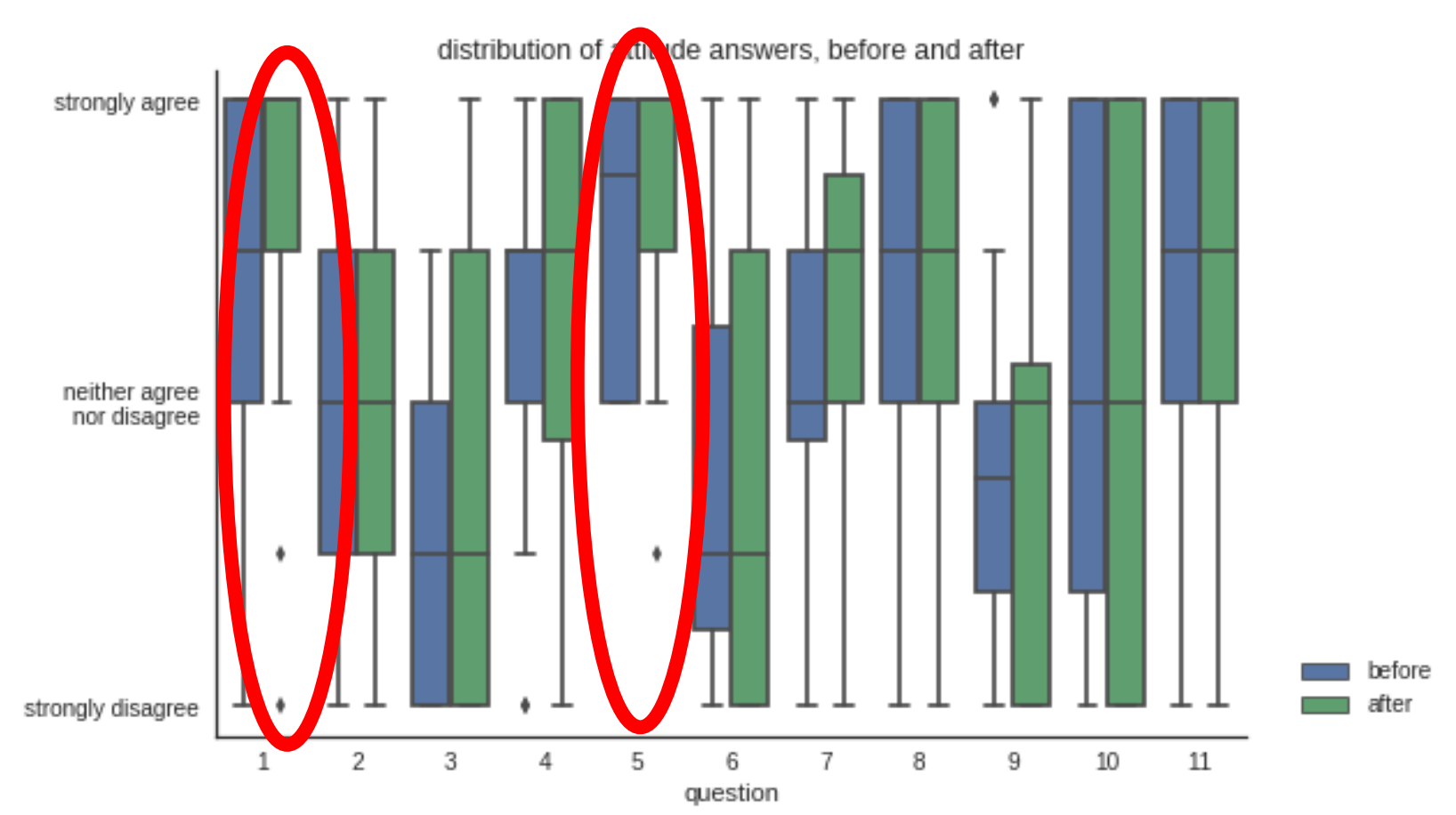

Q 1: Interpreters have a responsibility to help patients prepare for death $Q$ 5: Advance care planning is a basic responsibility of interpreters

Figure 2: Confidence in Palliative Care Conversations
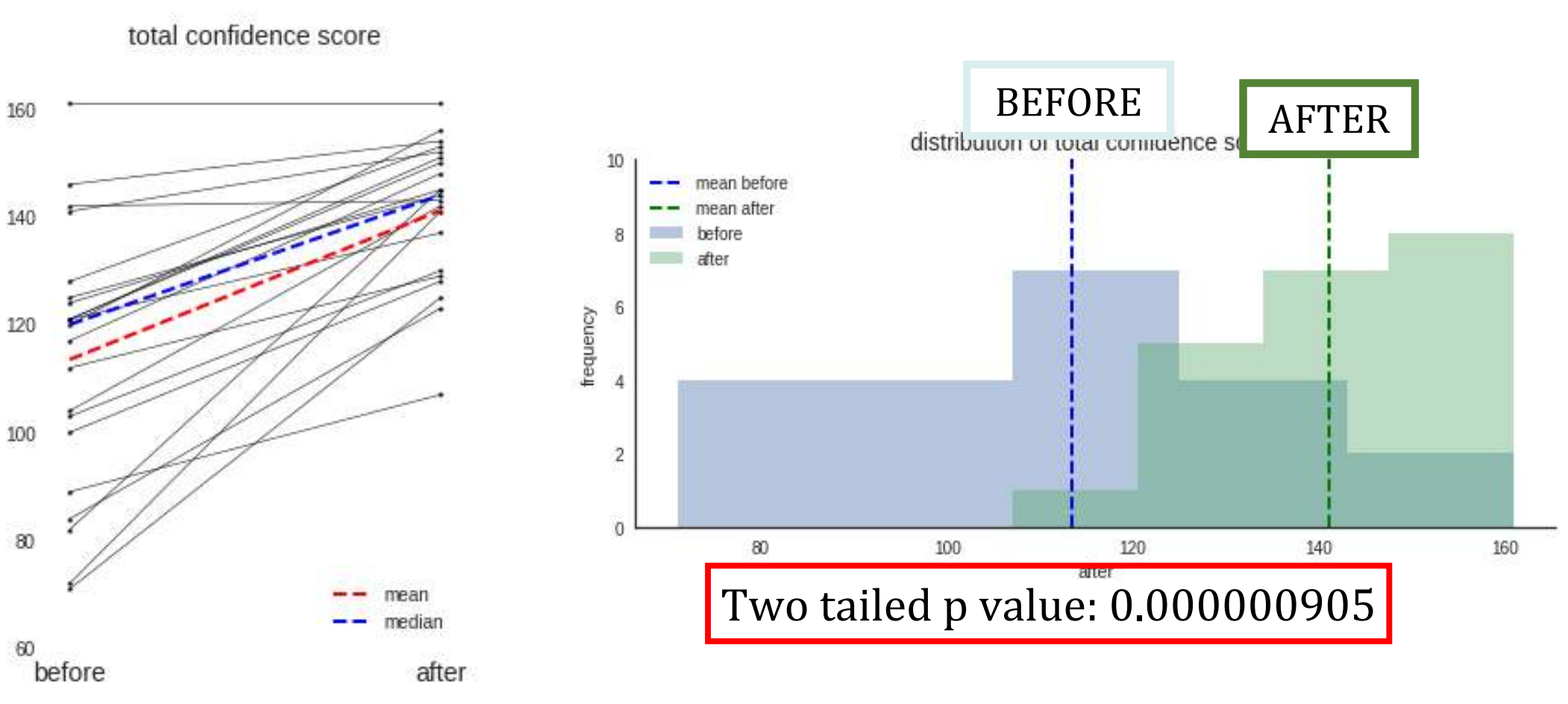

Acknowledgements: We thank our interpreter colleagues, Yilu Ma, MS, MA, CMI and Marta Solis, CMI for their invaluable input. 